\title{
Thiolated chitosan nanoparticles as a delivery system for antisense therapy: evaluation against EGFR in T47D breast cancer cells
}

\author{
Fatemeh Talaei' \\ Ebrahim Azizi ${ }^{2}$ \\ Rassoul Dinarvand ${ }^{3}$ \\ Fatemeh Atyabi ${ }^{3}$
}

'Novel Drug Delivery Systems Lab, ${ }^{2}$ Molecular Research Lab, Department of Pharmacology and Toxicology, ${ }^{3}$ Nanotechnology Research Centre, Faculty of Pharmacy, Tehran University of Medical Sciences, Tehran, Iran
Correspondence: Fatemeh Atyabi Nanotechnology Research Centre, Faculty of Pharmacy, Tehran University of Medical Sciences, Tehran I4I7I, Iran Tel +98 2I 66959052

Fax +98 21 66959052

Email atyabifa@tums.ac.ir
This article was published in the following Dove Press journal:

International Journal of Nanomedicine

13 September 2011

Number of times this article has been viewed

Abstract: Thiolated chitosan has high transfection and mucoadhesive properties We investigated the potential of two recently synthesized polymers: NAC-C (N-acetyl cysteine-chitosan) and NAP-C (N-acetyl penicillamine-chitosan) in anticancer drug delivery targeting epidermal growth factor receptor (EGFR). Doxorubicin (DOX) and antisense oligonucleotide (ASOND)-loaded polymer nanoparticles were prepared in water by a gelation process. Particle characterization, drug loading, and drug release were evaluated. To verify drug delivery efficiency in vitro experiments on a breast cancer cell line (T47D) were performed. EGFR gene and protein expression was analyzed by real time quantitative polymerase chain reaction and Western blotting, respectively. A loading percentage of $63 \% \pm 5 \%$ for ASOND and $70 \% \pm 5 \%$ for DOX was achieved. Drug release data after 15 hours showed that ASOND and DOX were completely released from chitosan-based particles while a lower and more sustained release of only $22 \% \pm 8 \%$ was measured for thiolated particles. In a cytosol simulated release medium/reducing environment, such as found intracellularly, polymer-based nanoparticles dissociated, liberating approximately $50 \%$ of both active substances within 7 hours. ASOND-loaded polymer nanoparticles had higher stability and high mucoadhesive properties. The ASOND-loaded thiolated particles significantly suppressed EGFR gene expression in T47D cells compared with ASOND-loaded chitosan particles and downregulated EGFR protein expression in cells. This study could facilitate future investigations into the functionality of NAP-C and NAC-C polymers as an efficient ASOND delivery system in vitro and in vivo.

Keywords: thiolated chitosan, nanoparticles, doxorubicin, antisense oligonucleotide, epidermal growth factor receptor, T47D breast cancer cells, controlled release

\section{Introduction}

As our understanding of the biological systems at molecular level increases, most of the biological expressions including onset of pathogenesis can be attributed more precisely to molecular dysfunctions. ${ }^{1}$ The promise of using antisense oligonucleotide (ASOND) to control gene function in diseased cells was first recognized over 20 years ago, ${ }^{2}$ but the major challenge in translating promise into clinical utility has been the lack of a method in delivering ASOND-based drugs to cells while avoiding the breakdown of these oligonucleotides through formulation or along the delivery routes. Thus, even though in vitro laboratory studies have been promising, the pace of developing this new class of drugs has been slow because of the difficulties mainly present in DNA/ RNA delivery to the cells. ${ }^{3}$ ASONDs are able to downregulate or specifically turn off individual genes and have therefore received much attention in gene therapy considering 
their relative ease of synthesis and use. ${ }^{4}$ In the fight against cancer, ASOND-based drugs that block cancer genes from producing malicious proteins have the potential to become a powerful new weapon to complement drugs that function in other different ways.

ASOND is quite effective in decreasing gene expression and protein production, a process known as gene knock down. ${ }^{5}$ Polymers and lipoplexes are two main vehicles with high potential to be used in ASOND drug delivery. Although $7.1 \%$ of the gene therapy clinical trials by March 2010 were based on lipoplexes, ${ }^{6}$ several problems continue to limit their application as drug products for human use. For instance, the formation of lipoplexes involves complex interactions between the lipid molecules, in addition to those with nucleic acids. Additionally, the ability to control the size and morphology of lipoplexes is rather limited, with resultant instability problems over time. ${ }^{7}$ Furthermore, toxicity associated with the use of lipoplexes in terms of immune stimulation of the host can also limit in vivo application. The toxicity of lipoplexes is closely associated with the administered dose and it may in part result from their large size and the high positive surface charge. ${ }^{8,9}$ According to these limitations cationic polymers are interesting alternatives to cationic lipids. Furthermore the self-assembly of polymer nanoparticles does not involve interactions of the polycation molecules with each other, resulting in better control of their physical properties compared with lipoplexes. The chemical structures of various polymers contain repeated units that can be easily manipulated by chemical modification to improve the physical and biological properties of the resultant polyplexes, and consequently enhance their transfection efficiency. ${ }^{10}$ Among the different properties studied in relation to nanoparticles, particle size and stability are the most critical issues that can affect the drug delivery process. According to this knowledge, a careful choice of nanoparticle formulation method is necessary in preparing particles which are loaded with different substances.

Epidermal growth factor receptor (EGFR) is a transmembrane receptor whose overexpression in breast cancer predicts poor prognosis and is inversely correlated with expression of estrogen receptor. ${ }^{11}$ T47D cells belong to a moderately malignant breast cancer cell line which is placed somewhere in between MCF7 and BT474 breast cancer cell lines according to EGFR expression level. ${ }^{12}$ The inhibition of EGFR expression in all three cell lines has been shown to significantly inhibit cancer growth; thus any of these cells could be chosen to assess the potential of EGFR inhibition using an anticancer formulation.
Owing to its proven cytocompatibility, biodegradability, low toxicity, and high cationic potential, chitosan is a suitable candidate as a drug carrier. ${ }^{13}$ Thiolated chitosan has a high level of cohesive-, mucoadhesive-, enzyme inhibitory-, and permeation-enhancing properties, ${ }^{14}$ In order to investigate and evaluate the various properties of two recently synthesized thiolated chitosan polymers, N-acetyl cysteine-chitosan (NAC-C) and N-acetyl penicillamine-chitosan (NAP-C), in anticancer drug delivery, these agents were formulated as nanoparticles loaded with ASOND and doxorubicin (DOX), which are known anticancer agents. This study further endeavored to establish the potential efficiency of these particles in an in vitro cancer model cell line known as T47D (human breast epithelial tumor cells) and evaluate the results.

\section{Materials and methods Preparation of thiolated chitosan}

To prepare low-molecular-weight chitosan (LMWC), $500 \mathrm{mg}$ of chitosan (medium molecular weight: $400 \mathrm{kDa}$; deacetylation degree $84.7 \%$; Fluka Germany) was dissolved in acetic acid $(6 \%[\mathrm{v} / \mathrm{v}])$. Sodium nitrite $\left(\mathrm{NaNO}_{2}, 20 \mathrm{mg}\right)$ dissolved in $2.5 \mathrm{~mL}$ demineralized water was added to the solution of polymer. The mixture was incubated for 1 hour under continuous stirring. Sodium hydroxide (4 M) was added to the mixture leading to $\mathrm{LMWC}$ precipitation at $\mathrm{pH}$ 9. The precipitate was filtered and washed with cold acetone. The residue was resolubilized in $3.5 \mathrm{~mL}$ of $0.1 \mathrm{M}$ acetic acid and exhaustively dialyzed against demineralized water using a dialysis tube (MW cut-off $12 \mathrm{kDa}$ ) followed by lyophilization at $-30^{\circ} \mathrm{C}$ and a pressure down to $0.01 \mathrm{mbar}$. The molecular weight of the hydrolyzed chitosan was determined by dynamic light scattering using a nanosizer instrument (Malvern Zetasizer laser, Malvern Instruments, UK).

Two previously synthesized and characterized thiolated chitosans were prepared. ${ }^{14}$ Due to its significant effect on formulation size, conformation, and drug delivery LMWC $(15 \mathrm{kDa})$ was used for thiolated chitosan preparation. ${ }^{15}$ Briefly, 300 mg EDAC (1-ethyl-3-(3-dimethyl-aminopropyl) was added to $500 \mathrm{mg}$ LMWC dissolved in $50 \mathrm{~mL}$ distilled water containing $2 \mathrm{~mL} \mathrm{HCl}(1 \mathrm{~N})$ and agitated for complete dissolution of EDAC. NAC or NAP (500 mg) was added to the mixture. The $\mathrm{pH}$ value of the preparations was adjusted to 5 and the mixtures were left at room temperature for 3 hours under continuous stirring and capped with nitrogen gas-filled balloons to prevent oxidation of thiol groups during the synthesis process. Other samples serving as controls were prepared by the same method excluding EDAC. To isolate 
the conjugated polymer and eliminate the unbound NAC or NAP, the mixtures were dialyzed for 3 consecutive days at $10^{\circ} \mathrm{C}$ in the dark using a dialysis tube (MW cut off $12 \mathrm{kDa}$ ) as follows: first against $1.4 \mathrm{mM}$ lactic acid, and then twice against the same medium but containing $1 \% \mathrm{NaCl}$ to reduce ionic interaction between the cationic polymer and the anionic sulfhydryl groups, followed by dialysis against $1.4 \mathrm{mM}$ lactic acid, and finally against $0.6 \mathrm{mM}$ lactic acid to adjust the $\mathrm{pH}$ to 4 . After 3 days the samples were lyophilized at $-30^{\circ} \mathrm{C}$ and stored at $4^{\circ} \mathrm{C}$ for further use.

\section{Cell toxicity assays}

A stable formulation should have a low cell toxicity with minimum effect on cell growth or viability. Roswell Park Memorial Institute culture medium (RPMI), containing salts, amino acids, and vitamins, was used as the T47D breast cancer cell medium in these experiments. RPMI 1640 (Gibco) culture medium supplemented with $10 \%$ fetal bovine serum (FBS) and $100 \mathrm{U} / \mathrm{mL}$ penicillin, $100 \mu \mathrm{g} / \mathrm{mL}$ streptomycin, $2 \mathrm{mmol} / \mathrm{L} \mathrm{L}$-glutamine, and $1 \mathrm{mmol} / \mathrm{L}$ sodium pyruvate (Invitrogen) was prepared under sterile conditions. The T47D culture medium was kept at $4^{\circ} \mathrm{C}$ and warmed to $37^{\circ} \mathrm{C}$ before use. A breast cancer wild type cell line, T47D, was obtained from the American Type Culture Collection. This differentiated epithelial cell line contains both estrogen and progesterone receptors with a medium level of EGFR expression compared with other breast cancer cell lines.

Stocks of synthesized polymers (NAC-C, NAP-C) and control (LMWC) with predetermined concentrations of 0.25 , $0.5,1.0$, and $2.0 \mathrm{mg} / \mathrm{mL}$ in acetate buffer ( $5 \mathrm{mM}$; pH 5.5) were prepared. These concentrations were obtained after the study of particle conformation and stability. In brief, $10 \mathrm{mg} / \mathrm{mL}$ stock concentrations of synthesized polymers (NAC-C, NAP-C) and control (LMWC) were used to prepare $0.25,0.5,1.0$, and $2.0 \mathrm{mg} / \mathrm{mL}$ solutions in a mixture containing dextrose $(1 \%)$ and sodium sulfate $(5 \mathrm{mM})$ at a ratio of $3: 1$. The preparations were then filtered using $0.22-\mu \mathrm{m}$ filters.

T47D cells were thawed and counted using the direct method of Trypan blue dye exclusion. ${ }^{16,17}$ The cells were plated at 10,000 cell per well in a 96-well culture plate using RPMI supplemented only with $10 \%$ FBS. The plates were left at $37^{\circ} \mathrm{C}$ inside a $5 \% \mathrm{CO}_{2}$ incubator. The medium of the wells containing cells with $80 \%$ confluence was replaced by $100 \mu \mathrm{L}$ of medium containing $12 \mu \mathrm{L}$ of each sample's concentrate preparation mixed with $88 \mu \mathrm{L}$ of free RPMI. The plates were kept inside the incubator at $37^{\circ} \mathrm{C}$ for 24 hours. The culture medium was then replaced with RPMI containing both FBS and antibiotic. The optical density (OD) of each well was measured by a solution composed of a novel tetrazolium compound [3-(4,5-dimethylthiazol-2-yl)-5-(3-carboxymet hoxyphenyl)-2-(4-sulfophenyl)-2H-tetrazolium, inner salt; MTS] and an electron coupling reagent (phenazine methosulfate). In this assay, known as MTS assay, $20 \mu \mathrm{L}$ of the ready to use solution is added to each well and the plates are placed at $37^{\circ} \mathrm{C}$ for 2 hours followed by the measurement of the absorption using a plate reader at $490 \mathrm{~nm}$ wavelength following promega protocol. ${ }^{18}$ The experiments were repeated three times and the data were collected each time from four wells to compare with control data (FBS-free RPMI).

\section{Preparation of drug-loaded nanoparticles and investigation of physical and toxicological properties}

A $2 \mathrm{mg} / \mathrm{mL}$ stock solution of DOX (Sigma D1515) in normal saline was mixed with RPMI to prepare a $2.5^{*} 10^{-7} \mathrm{M}$ solution. ASOND stock $(2.5 \mu \mathrm{L}$ of $100 \mu \mathrm{M})$ was mixed with $37.5 \mu \mathrm{L}$ dextrose $(1 \%)$ and $10 \mu \mathrm{L}$ sodium acetate buffer to a volume of $50 \mu \mathrm{L}$ (ASOND sequence; 5'-CCGTGGTCATGCTCC-3', complementary to positions 3811-3825 of the human EGFR cDNA, which contains the opal translation termination codon at residues 3817-3819). To prepare drug-loaded nanoparticles a concentration of $0.5 \mathrm{mg} / \mathrm{mL}$ polymer in sodium acetate buffer was prepared. Sodium sulfate $\left(\mathrm{Na}_{2} \mathrm{SO}_{4}\right.$, $5 \mu \mathrm{L}$ of $5 \mathrm{mM})$ was added to ASOND $(12 \mu \mathrm{L})$ and DOX $(180 \mu \mathrm{L})$ stock solutions and $2.5 \mu \mathrm{L}$ of the polymer stock solution was added to each tube. The spontaneously formed nanoparticles were vortexed for 50 seconds. ${ }^{19}$ Drug-loaded nanoparticles were recovered by centrifugation at 19,000 rpm for 30-45 minutes and washed three times with distilled water to obtain the final pellet, which was lyophilized and kept at $4^{\circ} \mathrm{C}$ for future experiments.

To study the stability of the particles, nanoparticles were exposed to acidic and basic media $(0.1 \mathrm{~N} \mathrm{HCl}$ and $0.01 \mathrm{~N}$ $\mathrm{NaOH}$ ). Nanoparticle formulations were stored in glass tubes covered with aluminum foil and left for 24 hours at two temperatures: $30 \pm 2{ }^{\circ} \mathrm{C}$ and $4 \pm 2{ }^{\circ} \mathrm{C}$. A vacuum chamber was used to remove the residual water. Different particle formulations were sputter-coated with a gold layer (sputter coater AGAR B7340, Standard, UK) and viewed with a scanning electron microscope (SEM; Philips XL30 Scanning microscope, Philips, the Netherlands) to assess morphological changes.

To assess the cell toxicity levels of the active substances and nanoparticle formulations, T47D cells were plated at 10,000 cells/well in 96-well plates. After reaching $80 \%$ confluence, the cells were brought into contact with 
$100 \mu \mathrm{L}$ of FBS-free RPMI containing above-mentioned concentrations of DOX, ASOND, and $0.5 \mathrm{mg} / \mathrm{mL}$ of nanoparticle formulations (NAC-C, NAP-C and LMWC, ASOND + NAC-C, ASOND + NAP-C, ASOND + LMWC, DOX + NAC-C, DOX + NAP-C and DOX + LMWC) for 24 hours at $37^{\circ} \mathrm{C}$. FBS-free and FBS-enriched RPMI were chosen as controls to exclude any chance of FBS interaction with cationic polymers. T47D cell survival was assessed by applying a standard MTS assay following the same procedure as explained in Cell toxicity assays section. The experiments were repeated three times and the data were collected each time from four wells and compared with control data.

\section{Drug-loading and drug-release studies}

The release of DOX and ASOND from nanospheres was monitored in the presence of $20 \mathrm{mM}$ sodium phosphate buffer $\mathrm{pH}$ 7. Nanoparticle solutions were filled into a $12 \mathrm{kDa}$ MW cut-off dialysis tube (Sigma-Aldrich, St. Louis, MO) and dialyzed against $1 \times$ PBS (phosphate buffer saline) for 15 hours at $37^{\circ} \mathrm{C}$. Dissolution medium $(1 \mathrm{~mL})$ was taken at different time points for analysis of drug concentration in solution. The amount was replaced each time by fresh PBS to prevent drug saturation. The oligonucleotide concentration in the medium was determined by comparing the UV absorbance values $(\lambda=260 \mathrm{~nm})$ before and after release..$^{20}$

The concentration of the DOX released into the medium was determined using a UV-spectrophotometer (UV spectrophotometer 1201, Shimadzu Co. Japan) at $488 \mathrm{~nm}^{21}$

To study the effects of a reducing environment on drug release from these particles, a simulated environment similar to that of cytosol was created according to Carlisle et $\mathrm{al}^{22}$ by the addition of $0.01 \%$ threo-1,4-dimercapto-2,3-butandiol (DTT) as a reducing agent to PBS medium replacing PBS in the above experiment. The release data in this medium were obtained and compared with PBS medium data.

Drug content and loading efficiency of nanoparticles were calculated by the following formulae:

Drug contents $=[($ drug weight in the nanoparticles $) /$ (weight of nanoparticles) $] \times 100$

Loading efficiency $=[$ (Residual drug in the nanoparticle)/(initial feeding amount of drug) $] \times 100$

\section{RT-PCR and SYBER green real time PCR}

T47D cells were seeded in FBS-free RPMI in a 96-well culture plate. Twenty-four hours later the medium over the cells was replaced with $100 \mu \mathrm{L}$ FBS free RPMI (control), polymeric nanoparticles \pm ASOND and DOX (ASOND + NAC-C,
NAC-C, DOX + NAC-C, NAP-C, ASOND + NAP-C, Dox + NAP-C, chitosan, ASOND + chitosan, DOX + chitosan), free ASOND and free DOX at concentrations previously mentioned (section Preparation of drug-loaded nanoparticles) All preparations were diluted in FBS-free RPMI to a final volume of $100 \mu \mathrm{L}$. The plates were incubated for 24 hours at $37^{\circ} \mathrm{C}$ and the culture medium was replaced by RPMI medium containing both FBS and antibiotic. Twenty-four hours later, the culture medium over the cells was emptied and $200 \mu \mathrm{L}$ of TRI reagent was added to each well. The plates were put on a shaker for 5 minutes and the content of each well was then emptied into a sterile RNase/DNase-free microtube. Chloroform $(120 \mu \mathrm{L})$ was added to each microtube. The tubes were vortexed for 15 seconds and incubated at room temperature for 10 minutes.

The samples were centrifuged at $11,000 \mathrm{rpm}$ to separate the different phases. Three phases were separated with the upper phase containing RNA. This phase was transferred to a clean microtube and $300 \mu \mathrm{L}$ of isopropanol was added to the tubes and mixed for 10 minutes at room temperature. The samples were then centrifuged at $4^{\circ} \mathrm{C}$ at $11,000 \mathrm{rpm}$ for 10 minutes. The above solution was emptied and $750 \mu \mathrm{L}$ ethanol 75\% (7.5 mL Merck ethanol and $2.5 \mathrm{~mL}$ RNase-free diethylpyrocarbonate [DEPC] water) was added to the white precipitate of RNA and mixed with a vortex instrument to wash the RNA residual. These samples were then centrifuged at $4^{\circ} \mathrm{C}$ and $11,000 \mathrm{rpm}$ for 7 minutes. The above solution was emptied and the RNA precipitate was left to dry at room temperature. The concentration and quality of the RNA samples were investigated using a spectrophotometer. The precipitated RNA was diluted in $50 \mu \mathrm{L}$ of DEPC water and transferred into a fresh microtube. The measured OD was incorporated into the following formula: $[\mathrm{RNA}](\mu \mathrm{g} / \mathrm{mL})=\mathrm{A} 260 \times$ Dilution Coefficient $\times 40$.

The purity of RNA was determined by calculating OD $260 / 280$ ratio which was measured as $\sim 1.9$, indicating the absence of protein or phenol contaminants. The RNA samples were stored at $-20^{\circ} \mathrm{C}$.

RevertAid $^{\mathrm{TM}}$ First Strand cDNA Synthesis kit was used to make cDNA from RNA extracts according to the protocol provided by manufacturer. ${ }^{23}$ The product of RT was transferred to $-20^{\circ} \mathrm{C}$ for later use in real time QPCR (quantitative polymerase chain reaction).

Specific EGFR primers were used to amplify the EGFR sequence in cDNA. EGFR primers [(forward) I (18-mer): 5'-CAACATCTCCGAAAGCCA, and (reverse) II (19-mer): 5'-CGGAACTTTGGGCGACTAT] and $\beta$-actin primers [(forward), 5'-GTCCTGTGGCATCCATCCACGAAACT 
and (reverse), 5'-TACTTGCGCTCAGGAGGAGCAA] were provided by TIB MOLBIOL Company.

SYBR green real time PCR was performed using Bio-Rad CFX384 C1000 and analyzed using Bio-Rad CFX manager 2.0 with reagents from the SYBR green PCR kit (QIAgen). After the addition of all components for each sample into the PCR 384 well plate with each well containing a final volume of $10 \mu \mathrm{L}$, the samples were transferred to a thermal cycler and PCR was started as follows: predenaturation at $94^{\circ} \mathrm{C}$ (5 minutes; one cycle) followed by 35 cycles of denaturation at $94^{\circ} \mathrm{C}$ (30 seconds), annealing at $57^{\circ} \mathrm{C}$ (30 seconds), extension at $72^{\circ} \mathrm{C}$ ( 45 seconds), and a final extension at $72^{\circ} \mathrm{C}$ ( 8 minutes; one cycle), followed by a melting curve. All $\mathrm{Ct}$ (cycle threshold) values were collected at the exponential phase of the real time QPCR.

\section{Evaluation of EGFR protein expression}

T47D cells were seeded in FBS containing RPMI in sixwell culture plates. Twenty-four hours later the medium over the cells was emptied and PBS was used to wash the cells. The same groups (section RT-PCR and SYBER Green real time PCR) were prepared with appropriate polymer and drug concentrations in a volume of $1 \mathrm{~mL}$ FBS-free RPMI for each well. The plates were incubated for 24 hours at $37^{\circ} \mathrm{C}$ and the culture medium was replaced with RPMI medium containing both FBS and antibiotic. Forty-eight hours later, the culture medium over the cells was emptied and the treated and untreated T47D cells in six-well plates were washed with PBS and lysed in $120 \mu \mathrm{L}$ RIPA buffer (1 mM EDTA, 1\% Triton X-100, 0.1\% sodium deoxycholate, $0.1 \%$ sodium dodecyl sulfate, $140 \mathrm{mM}$ $\mathrm{NaCl}$ ) supplemented with protease inhibitor on ice. The protein concentration was measured by Bradford assay in all the samples. Loading buffer $(20 \mu \mathrm{L})$ was added to every $50 \mu \mathrm{g}$ of cell protein and run at $100 \mathrm{~V}$ for 70 minutes. Proteins were transferred to a nitrocellulose membrane and incubated with EGFR antibody (1:1000) overnight. The next day the membranes were washed and incubated with the secondary antibody for 2 hours at room temperature. Expression of EGFR (170 kDa) was detected by West Pico Chemiluminescent Substrate (SuperSignal, Thermo Scientific), photographed, and analyzed with genetool software (version 3.08, SynGene).

For immunocytochemical examination of T47D cells, cells were cultured on round glass cover-slips and treated as previously described. After the treatment cells were washed with PBS and fixed by acetone (100\%) for 10 minutes at room temperature, washed and rehydrated with PBS.
Hydrogen peroxidase activity was blocked by hydrogen peroxide (1\%) in PBS for 30 minutes and the slides were washed in PBS three times for 5 minutes each and incubated for 1 hour with 1\% primary antibody to EGFR (rabbit, Santa Cruz SC-03) in PBS containing 1\% bovine serum albumin (BSA) for 1 hour at room temperature, washed in PBS three times and incubated with $1 \%$ secondary antibody Goat AntiRabbit HRP (Dako, po448) in PBS containing $1 \%$ BSA for 1 hour at room temperature and again washed in PBS three times to remove the unbound antibodies. The signal was amplified by $1 \%$ of a third antibody, rabbit Anti Goat (Dako, po449) in PBS containing 1\% BSA. The slides were washed in PBS and Dako AEC + High Sensitivity Substrate Chromogen (k3469) was used in all stainings to visualize protein bond antibody. The nuclei were visualized by hematoxylin counterstaining blue staining of the cell nuclei. The slides were washed in water and covered by glass cover-slips and photographed at $40 \times$ resolution using a light microscope and camera.

\section{Statistical data analysis}

Statistical analysis was performed using GraphPad Prism version 5.00 for Windows (GraphPad Software, San Diego CA). ANOVA was used with Tukey test to compare the data groups with control.

\section{Results and discussion Synthesis of chitosan conjugates}

LMWC with a molecular weight of $15 \mathrm{kDa}$ and $100 \%$ solubility at $\mathrm{pH} 7$ was prepared. NAC and NAP attached covalently to the primary amino groups of chitosan via formation of amino bonds. The final structures are shown in Figure 1. EDAC (carbodiimide) activated the carboxylic acid moieties of NAC and NAP. LMWC was treated by the same procedure omitting EDAC during the coupling reaction and used as control. The lyophilized NAC-C and NAP-C appeared as white, odorless powders of fibrous structure that swelled easily at $\mathrm{pH}<8$ and formed a transparent gel of high viscosity. The lyophilized conjugates were stored at $4^{\circ} \mathrm{C}$. The characterization of these polymers has been already reported in our previous study. ${ }^{14}$ The amount of thiol groups grafted on chitosan was determined via iodometric titration at $\mathrm{pH} 1-2$ (1 mM iodine; indicator: starch). It was estimated that the highest amount of grafted thiol groups was obtained by the addition of $300 \mathrm{mg}$ EDAC at $\mathrm{pH}$ 5, with immobilization of $300 \mu \mathrm{mol}$ of thiol groups per gram chitosan. In the following experiments only polymers obtained from this optimum condition were used. 


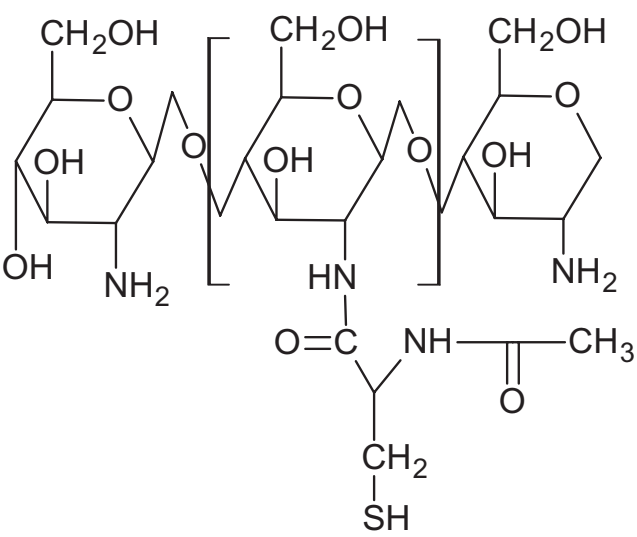

A



B

Figure I Presumptive structures of (A) NAC-chitosan and (B) NAP-chitosan conjugates. Abbreviations: NAC, N-acetyl cysteine; NAP, N-acetyl penicillamine.

\section{Cell toxicity assay}

For toxicological evaluation, different concentrations of polymers were tested on T47D cells and the level of cell proliferation was compared with FBS-free RPMI as control by MTS assay. The results indicate that all polymers have a low but significant degree of toxicity compared with FBS-free RPMI. Thiolated chitosan polymers did not show a higher level of toxicity than LMWC (Figure 2). The lower the concentration of polymers the more similar are the measured effect they have on the wells containing T47D cells, enabling us to prevent variation in measurements. There was a significant difference in cell proliferation with polymer concentrations compared with FBS-free RPMI. According to the results achieved in cell toxicity studies, the $0.5 \mathrm{mg} / \mathrm{mL}$ concentration of the polymers was chosen to perform future experiments. Polymers in this concentration formed the desired spherical

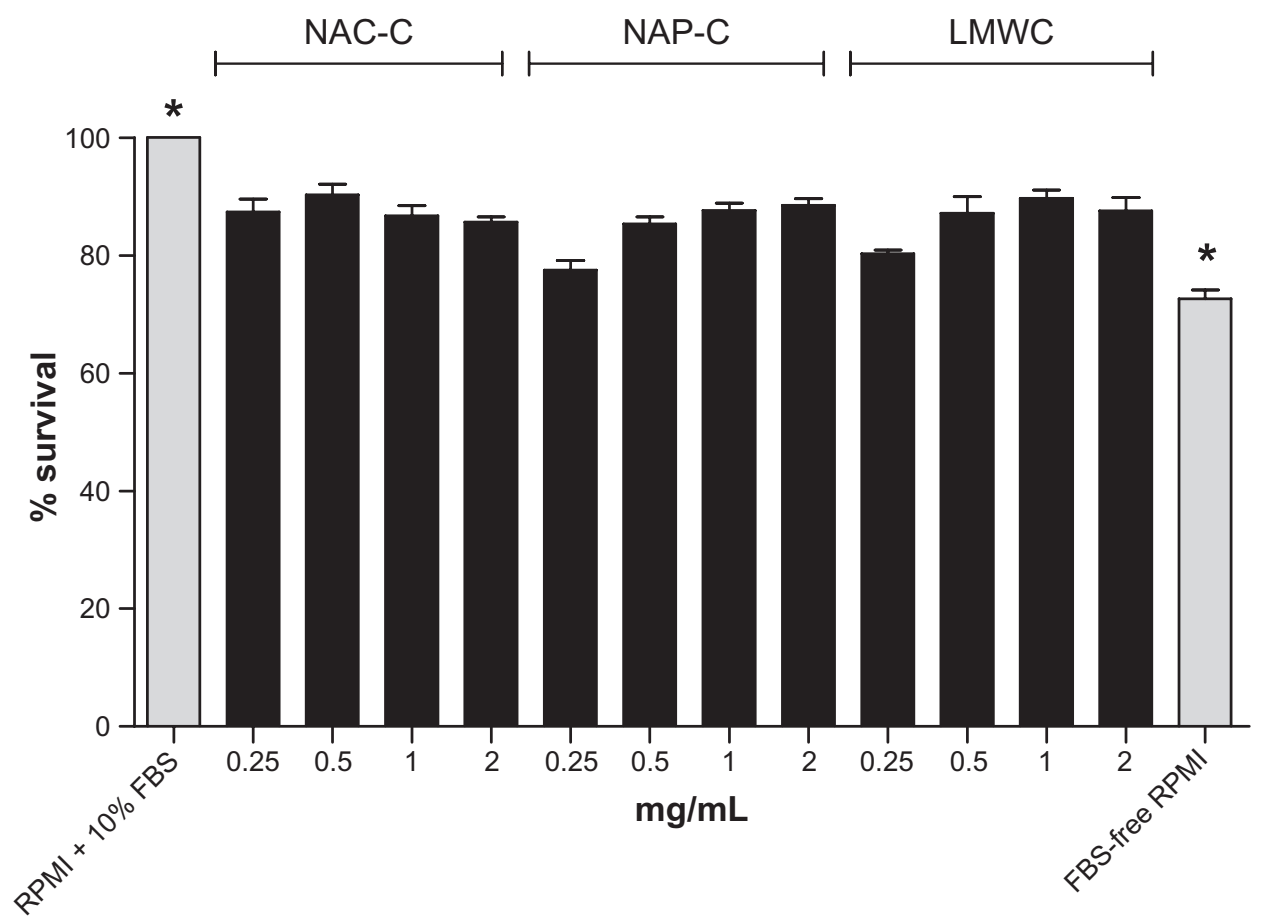

Figure 2 The effect of different concentration $(0.25,0.5,1,2 \mathrm{mg} / \mathrm{mL})$ of polymers (NAC-C, NAP-C, LMWC) on T47D cancer cell proliferation 48 hours after treatment compared with positive control (RPMI $+10 \%$ FBS) and negative control (FBS-free RPMI). The data are mean \pm SD of three experiments in four wells. In ANOVA tests all the data were compared to controls demonstrating a significant difference $(P<0.000 \mathrm{I})$.

Note: *Significantly different from all other groups.

Abbreviations: ANOVA, analysis of variance; NAC-C, N-acetyl cysteine-chitosan; NAP-C, N-acetyl penicillamine-chitosan; LMWC, low-molecular-weight chitosan; RPMI, Roswell Park Memorial Institute medium; FBS, fetal bovine serum; SD, standard deviation. 
nanoparticles with a diameter of 150-300 nm and according to the results obtained later on, were able to capture and carry active substances to the desired site. ANOVA was used with Tukey test to compare the data groups to control. All the data groups showed a significant difference compared to control $(P<0.0001)$.

\section{Preparation of nanoparticles and nanoparticle characterization}

Nanoparticles containing DOX and ASOND were prepared following the procedure explained in section Preparation of drug-loaded nanoparticles. The drug-loaded nanoparticles were centrifuged at high speed, the supernatant was emptied, and the remaining nanoparticles were lyophilized. Polymer nanoparticles without any drug were also prepared to make a comparison with those containing the active substances. The SEM micrograph from four different formulations are shown in Figure 3 (A-D); 80\%-90\% of the particles had a size range of 200-300 nm with a spherical shape.

These nanoparticles were dispersed in phosphate buffer ( $\mathrm{pH}$ 7.4) and analyzed based on their surface charge and size with a sharp peak at the indicated points (Table 1), indicating a $55 \pm 5 \%$ particle size distribution frequency (data not shown). Thiolated chitosan particles had a higher zeta potential than chitosan but zeta potential decreased significantly for nanoparticles containing ASOND. The lower zeta potential obtained for ASOND-containing particles is probably due to the negative charge of the active substance. ${ }^{24}$

In particles containing DOX the zeta potential of the particles slightly increased due to the cationic nature of this substance. ${ }^{25}$ The particle size for all formulations remained in the range $200-230 \mathrm{~nm}$. The size range of these particles is comparable to the particle sizes obtained by other groups. ${ }^{26}$

Cell toxicity assay conducted according to MTS where active substances and formulated nanoparticles were used demonstrated that DOX in any form (free/nanoparticle) applied to cells was toxic and significantly decreased cell survival in comparison with both controls (RPMI $+10 \%$ FBS and FBS-free RPMI) and other treatments (Figure 4). Thus the cells were not resistant to this compound. Thiolated nanoparticles containing ASOND showed lower cytotoxic effects than free ASOND thus signifying a lower toxicity of ASOND in nanparticle formulation. NAC-C and NAP-C did not show higher cell toxicity levels than chitosan and FBS-free RPMI (negative control.) All the treatments showed a significant decrease in cell survival compared with RPMI $+10 \%$ FBS (positive control). FBS-enriched RPMI medium had the highest growth-inducing effect on cells and DOX-containing medium showed the highest inhibitory effect on cell proliferation.

\section{Drug-loading and drug-release studies}

The thiolated nanoparticles had a loading efficiency of $63 \%$ for ASOND and 70\% for DOX. LMWC nanoparticles containing ASOND or DOX had a 50\%-60\% loading efficiency. ASOND had a negative charge, probably forming a stronger binding force to the positively charged polymers like thiolated chitosan. Entrapment of DOX, a cationic and hydrophilic molecule, into nanoparticles formed by ionic gelation of the positively charged polysaccharides, chitosan/thiolated chitosan, was achieved successfully probably due to the addition of $\mathrm{Na}_{2} \mathrm{SO}_{4}$ directly to this substance as it contributes to the decreasing water solubility of DOX. ${ }^{27}$ In examining thiolated chitosan particles exposed to either acidic or basic media $(0.1 \mathrm{~N} \mathrm{HCl}$ and $0.01 \mathrm{~N} \mathrm{NaOH})$ no particular change was observed according to SEM graphs, demonstrating the stability in both media. The release of drug from particles was examined in PBS ( $\mathrm{pH}$ 7.4). In a second experiment the release studies were performed under reducing conditions achieved by $0.01 \%$ DTT, simulating intracellular redox conditions of cytosol as described previously by Carlisle et al. ${ }^{28}$ There was a controlled release pattern during 15 hours for all the
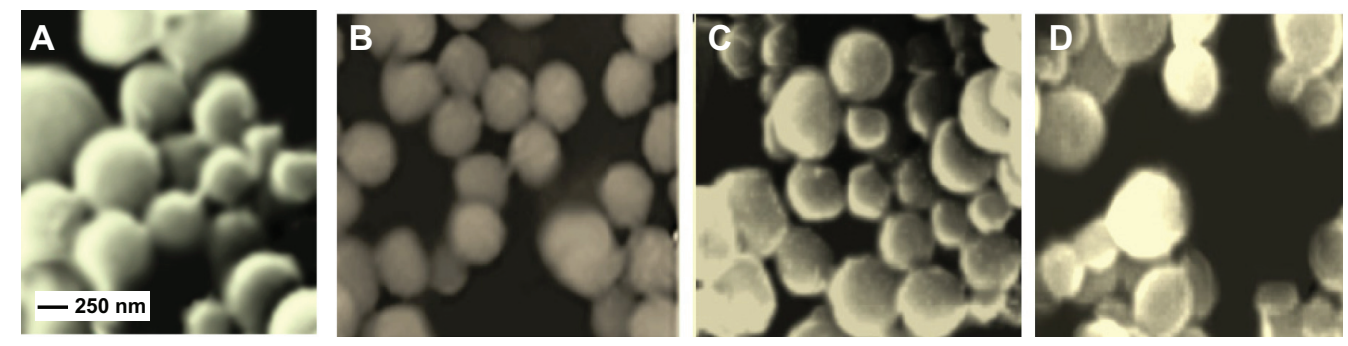

Figure 3 Representative SEM micrograph of nanoparticles formulated via sulfate gelation with $\mathrm{Na}_{2} \mathrm{SO}_{4}$. (A) chitosan nanoparticles, (B) thiolated chitosan (NAP-C) nanoparticles, (C) DOX-NAC-C nanoparticles, (D) ASOND-NAC-C nanoparticles demonstrate a spherical, uniform shape with a particle size of I50-300 nm.

Abbreviations: DOX, doxorubicin; NAC-C, N-acetyl cysteine-chitosan; NAP-C, N-acetyl penicillamine-chitosan; ASOND, antisense oligonucleotide; SEM, scanning electron microscope. 
Table I The surface charge and size of different nanoparticle formulations was measured by a zeta sizer instrument in phosphate buffer

\begin{tabular}{lll}
\hline $\begin{array}{l}\text { Nanoparticle } \\
\text { formulations }(\mathbf{N}=\mathbf{3})\end{array}$ & $\begin{array}{l}\text { Zeta potential } \\
(\mathbf{m V})\end{array}$ & $\begin{array}{l}\text { Nano particle } \\
\text { size }(\mathbf{n m})\end{array}$ \\
\hline NAC-C-ASOND & $14 \pm 3$ & $224 \pm 5$ \\
NAP-C-ASOND & $16 \pm 2$ & $201 \pm 10$ \\
Chitosan-ASOND & $12 \pm 2$ & $264 \pm 5$ \\
NAP-C-DOX & $20 \pm 3$ & $275 \pm 5$ \\
NAC-C-DOX & $20 \pm 3$ & $326 \pm 5$ \\
Chitosan-DOX & $21 \pm 2$ & $227 \pm 5$ \\
NAC-C & $23 \pm 3$ & $214 \pm 10$ \\
NAP-C & $22 \pm 4$ & $188 \pm 5$ \\
Chitosan & $18 \pm 2$ & $224 \pm 5$ \\
\hline
\end{tabular}

Abbreviations: ASOND, antisense oligonucleotide; DOX, doxorubicin; NAC-C, $\mathrm{N}$-acetyl cysteine-chitosan; NAP-C, N-acetyl penicillamine-chitosan.

Notes: Data represent the mean of three replicates in two separate experiments (mean \pm SD).

particles (Figure 5A and B). Chitosan nanoparticles tended to have a faster rate of release probably because nanoparticles are less stable due to the lack of disulfide bonds compared with thiolated chitosan. Within 15 hours, ASOND and DOX were completely released from chitosan-based particles while only about $22 \%$ of drug was released from thiolated chitosan-based particles. These nanoparticles dissociated, liberating approximately $50 \%$ of both compounds within 7 hours. Thiolated chitosan nanoparticles were very stable, having formed inter-/intra-molecular disulfide bonds and decreasing drug release compared with chitosan particles. A reducing agent like DTT breaks up such bonds helping the release of drug from thiolated nanoparticles, a process similar to what happens inside a cell (Figure $5 \mathrm{~A}$ and B). The simulated medium did not affect drug release from chitosan nanoparticles and the data were not included in the graph.

\section{Analysis of EGFR gene expression in T47D cells}

T47D cells were brought into contact with different formulations. RNA was extracted from the cells and DNA was synthesized from the sequence. The DNA obtained from the reaction was amplified using specific primers against EGFR and $\beta$-actin by RT QPCR. The data were collected at the exponential phase of the reaction. The EGFR Ct value was normalized to $\beta$-actin (house keeping gene) and the relative expression for each sample was measured. Figure 6 shows the percentage knock down of EGFR in different treatments compared with FBS-free RPMI-treated cells ( $0 \%$ knock down.)

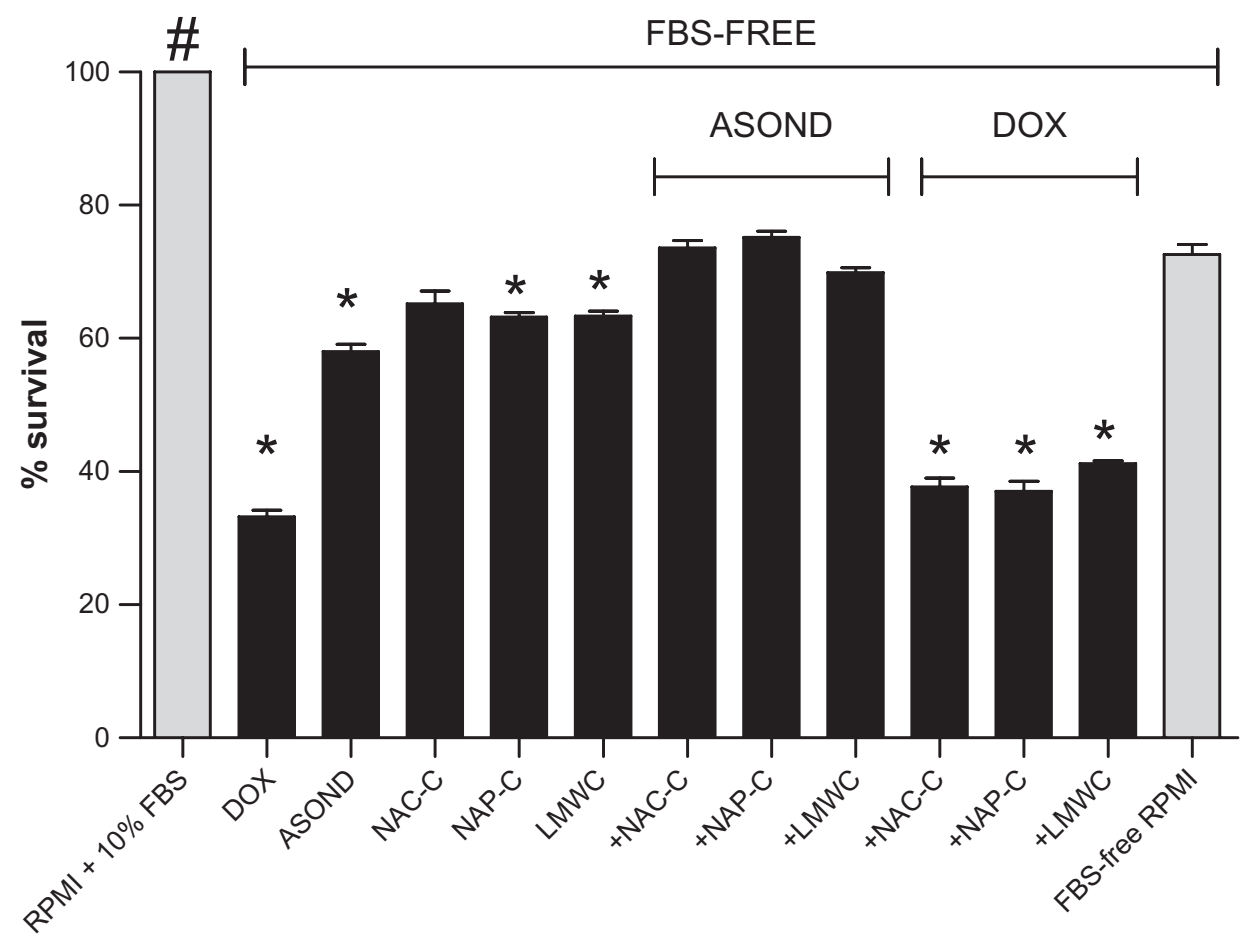

Figure 4 MTS assay measured the effect of polymer nanoparticles $(0.5 \mathrm{mg} / \mathrm{mL})$ containing ASOND and DOX $\left(25 \times 10^{-7} \mathrm{M}\right)$ on T47D cancer cell proliferation 24 hours after treatment. The data are mean \pm SD of three separate experiments in four wells. In ANOVA tests all the data were compared with controls (RPMI + I0\% FBS- and FBS-free RPMI). Notes: *Significantly different from FBS-free RPMI; \#RPMI + I0\% FBS is significantly different from all the other treatments $(P<0.000 \mathrm{I})$.

Abbreviations: ASOND, antisense oligonucleotide; DOX, doxorubicin; MTS, 3-(4,5-dimethylthiazol-2-yl)-5-(3-carboxymethoxyphenyl)-2-(4-sulfophenyl)-2H-tetrazolium, NAC-C, N-acetyl cysteine-chitosan; NAP-C, N-acetyl penicillamine-chitosan; LMWC, low-molecular-weight chitosan; RPMI, Roswell Park Memorial Institute medium; FBS, fetal bovine serum; SD, standard deviation. 

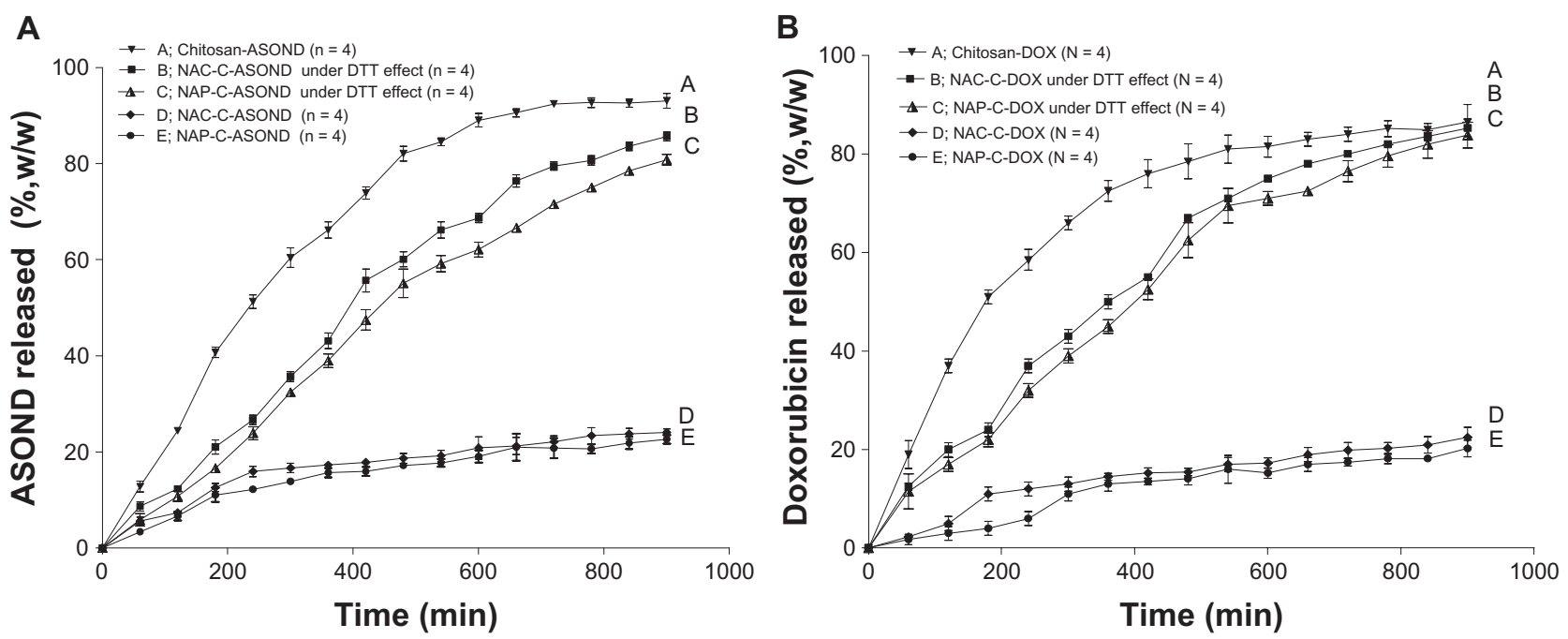

Figure 5 Percentage release of ASOND and doxorubicin from $5 \mathrm{mg}$ of drug-loaded nanoparticles during 15 hours in PBS and cytosol simulated medium using DTT. (A) Percentage release of ASOND, (B) Percentage release of doxorubicin. The release data are mean \pm SD for each formulation at different time points indicated $(n=4)$. Abbreviations: ASOND, antisense oligonucleotide; DOX, doxorubicin; NAC-C, N-acetyl cysteine-chitosan; NAP-C, N-acetyl penicillamine-chitosan; LMWC, lowmolecular-weight chitosan; RPMI, Roswell Park Memorial Institute medium; FBS, fetal bovine serum; PBS, phosphate buffered saline; DTT, threo-I,4-dimercapto-2,3butandiol; SD, standard deviation.

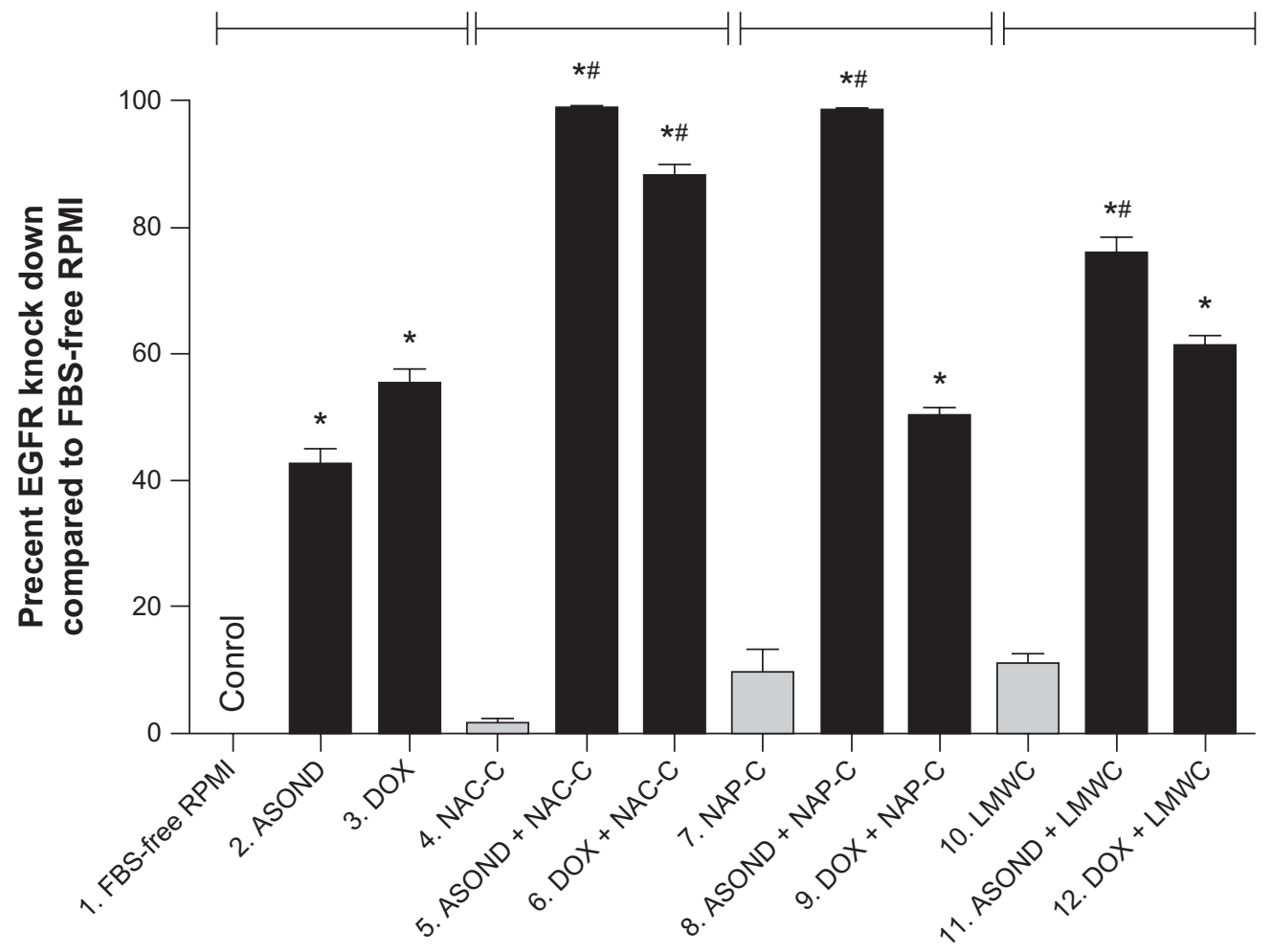

Figure 6 Real-time PCR analysis of EGFR gene expression in T47D cells subjected to different treatments. The results were normalized to beta-actin gene expression and the relative expression of EGFR was presented as percent knock down of EGFR. FBS-free RPMI treatment was chosen as control, which presented no knock down of EGFR and all the other cell treatments were compared with this control. All data are presented as percent knock down of EGFR and are significantly different to control.

Notes: *Difference to control or free polymers (gray bars) within each group separated by indicator lines; " significant difference of each formulation containing ASOND or DOX compared to free DOX or ASOND $(P<0.05)$; data are mean SD $(n=3)$.

Abbreviations: ASOND, antisense oligonucleotide; DOX, doxorubicin; EGFR, epidermal growth factor receptor; NAC-C, N-acetyl cysteine-chitosan; NAP-C, N-acetyl penicillamine-chitosan; LMWC, low-molecular-weight chitosan; RPMI, Roswell Park Memorial Institute medium; FBS, fetal bovine serum; PBS, phosphate buffered saline; SD, standard deviation. 


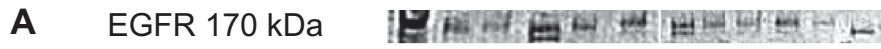

$\beta$-actin $42 \mathrm{kDa} \quad=-\pi-2-\pi$
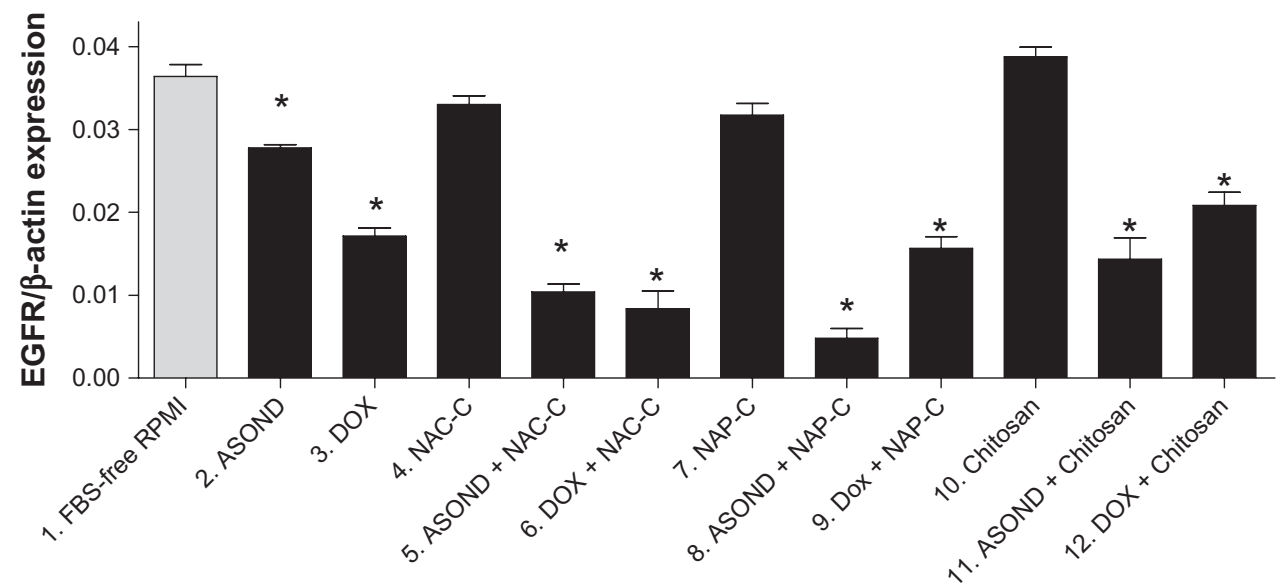

B
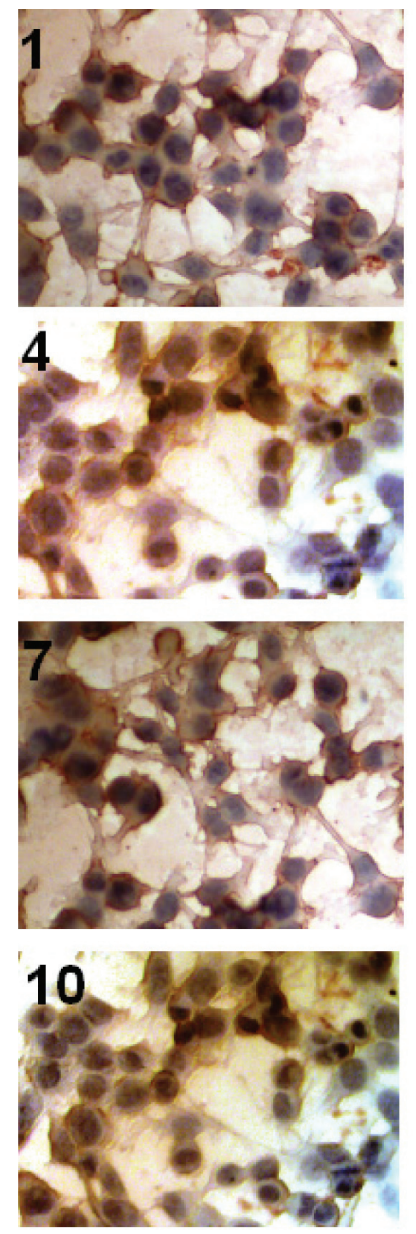
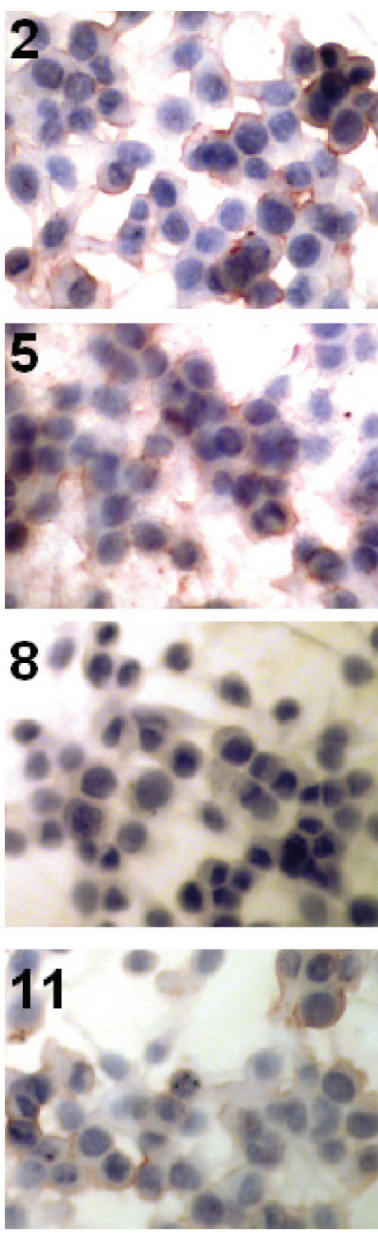
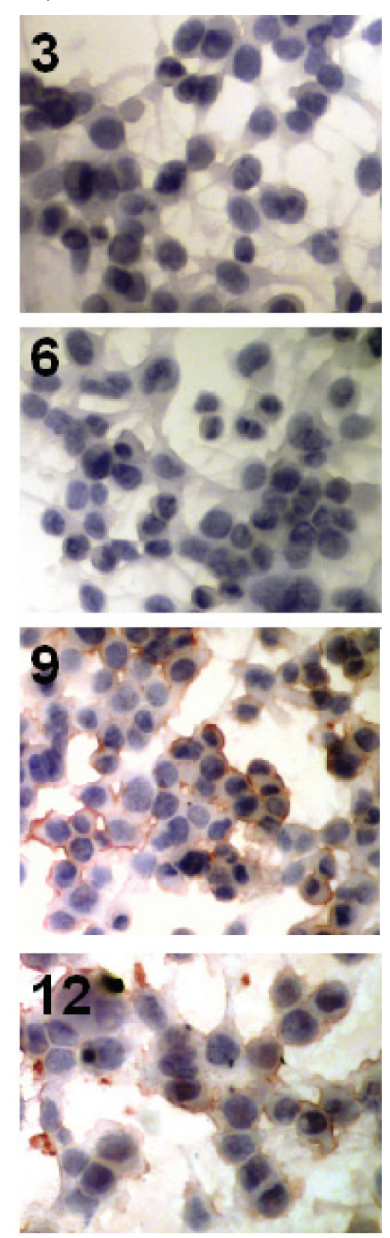

Figure 7 Analysis of EGFR protein expression in T47D cells. (A) Western blot analysis of EGFR expression in T47D cells through different treatments; (B) immunocytochemical analysis of EGFR protein expression in T47D cells. (I) FBS-free RPMI; (2) ASOND; (3) DOX; (4) NAC-C; (5) ASOND + NAC-C; (6) DOX + NAC-C; (7) NAP-C; (8) ASOND + NAP-C; (9) DOX + NAP-C; (10) Chitosan; (I I) ASOND + chitosan; (12) DOX + chitosan. The brown stain signifies higher expression of this protein which is observed in cytoplasm and on the membrane of the cells and the blue stain indicates the nuclei of the cell.

Note: *Significantly different from FBS-free RPMI treated cells as controls (mean $\pm S D ; n=3$ ).

Abbreviations: ASOND, antisense oligonucleotide; DOX, doxorubicin; EGFR, epidermal growth factor receptor; NAC-C, N-acetyl cysteine-chitosan; NAP-C, N-acetyl penicillamine-chitosan; LMWC, low-molecular-weight chitosan; RPMI, Roswell Park Memorial Institute medium; FBS, fetal bovine serum; PBS, phosphate buffered saline; SD, standard deviation. 
DOX and DOX-containing nanoparticles decreased EGFR expression significantly (Figure 6 [3, 6, 9, 12]) compared with control particles (Figure $6[1,4,7,10]$ ). DOX decreased EGFR expression more than ASOND. Probably this observation is due to DOX cytotoxic effects on T47D cells. In particular it was observed from cell survival data and EGFR expression level that thiolated chitosan polymers cannot decrease the cell toxicity of this compound. It is clear that because of the high level of cytotoxicity observed with this compound and the mechanism of action, DOX is not the preferred drug in EGFR suppression studies causing high levels of cell death.

ASOND (Figure 6 [2]) decreased the expression of EGFR twice as much as control, while thiolated nanoparticles containing ASOND (Figure $6[5,8,11]$ ) were twice as effective than ASOND or ASOND + LMWC (Figure 6 [11]) nanoparticles in EGFR knock down. From drug release, cell survival, and gene expression data it could be concluded that thiolated chitosan nanoparticles containing ASOND decreased the cytotoxic effects of this compound probably due to a slower rate of release, resulting in higher levels of EGFR gene suppression. EGFR expression level was confirmed by gel electrophoresis on the end product of RT QPCR (data not shown).

\section{Analysis of EGFR protein expression in T47D cells}

EGFR expression in T47D cells after different treatments was studied using Western blot analysis and cell antibody staining. Western blot and immunocytochemical results showed a significant downregulation of this protein in all treated groups except those treated with FBS-free RPMI, NAC-C, NAP-C, and chitosan (Figure 7A and B). The results show that ASOND-loaded particles (Figure 7A [5, 8, 11]) downregulated EGFR expression approximately twice as much as free ASOND (Figure 7A [2]). DOXloaded nanoparticles (Figure 7A $[6,9,12]$ ) showed a similar result to free DOX (Figure 7A [3]). Thus EGFR protein expression data further confirm EGFR gene expression data obtained through different treatments. Cell staining using EGFR antibody was performed. The control cells and cells treated with empty particles showed expression of EGFR protein in cytoplasmic portion and the membrane of the cells (Figure 7B [1, 4, 7, 10]). The intensity of the stain decreased through treatment with either free DOX or ASOND (Figure 7B $[2,5]$ ) and the expression was very low when nanoparticles loaded with DOX and ASOND were incorporated (Figure 7B [3, 6, 8, 9, 11]). Normally EGFR protein present on the cell membrane is internalized after activation of this protein which signifies the binding of this receptor to its substrate and activation of EGFR. ${ }^{29}$ While in T47D cells EGFR was observed in cytoplasmic and membrane portion of the cells, the localization of this protein after treatment was mostly on the cell membrane, which could signify a lower degree of expression due to either EGFR knock down by ASOND or inhibition of production, leading to lower functionality of this protein shown by a lower internalization level.

\section{Conclusion}

In this study the anticancer drugs ASOND and DOX were loaded into two recently synthesized thiolated chitosan polymers, NAC-C and NAP-C, by a gelation method. The efficiency of nanoparticle formulations against EGFR expression was evaluated in vitro on T47D breast cancer cells, with promising results.

The particle size and morphology of polymer nanoparticles depend on the structure and molecular weight of polymer, $\mathrm{pH}$ and ionic strength of the surrounding environment, method of preparation, and the charge of the polymer used for nanoparticle formulation. Thiolated chitosan under optimum conditions physically condenses nucleic acids. ${ }^{30}$ This contributes to the formation of nanoparticles $(200 \pm 30 \mathrm{~nm})$, with an acceptable level of cellular uptake demonstrated by downregulation of EGFR expression in T47D cells.

Different groups have investigated the potential benefits of ASOND-loaded chitosan nanoparticles in different settings. Ozbas-Turan et al have shown the potential of chitosan as a carrier of ASOND across the skin barrier to inhibit $\beta$-Gal with a long and sustained effect. The high permeability of ASOND into skin layer was noted to be probably due to the effect of chitosan on tight junctions and an increase in permeability. ${ }^{31}$ Manchanda et al have described that controlled-size chitosan nanoparticles produced using reverse micellar system is an efficient biocompatible ASOND delivery system with high cellular viability compared with lipofectamine. ${ }^{32}$ It has also been shown that chitosan nanoparticles protect the ASOND molecule during preparation and treatment. ${ }^{33}$ Protection of DNA from enzymatic degradation through these nanoparticles, which is critical for in vivo efficiency and a highly targeted delivery, is another known beneficial effect observed. ${ }^{34}$ Despited all the positive and promising results published, the use of these particles in in vivo studies is still limited. Thus, 
more in vitro studies are needed to further evaluate the potential use of such particles systematically in vivo.

The presence of thiol groups on the surface of the thiolated chitosan polymers (NAC-C and NAP-C) confers high mucoadhesive properties to the polymer, and the covalent cross linkage between sulfhydryl groups present on thiolated chitosan contributes to the formation of uniform nanoparticles stable in both acidic and basic media, as previously shown by our group. ${ }^{14}$

Recently Gazori et al investigated the significant inhibitory effect of ASOND-loaded chitosan/alginate nanoparticles on EGFR expression in T47D cells, ${ }^{35}$ which show the ASOND protective properties of chitosan polymer while creating a sustained release delivery system for ASOND and DOX. Here we show that the use of thiolated chitosan also prevents the degradation of the active substances promoting sustained release properties. According to the results obtained from our loading/release studies and resistance to acidic and basic media, NAC-C and NAP-C particles are able to protect and release the active substances within a set period of time, leading to a significant decrease in the expression level of EGFR.

In comparison with chitosan, thiolated chitosan nanoparticles gain triggered release properties in reducing environments and have a higher degree of structural stability and mucoadhesiveness. These particles have an acceptable drug-loading/capture capacity and show a significantly higher rate of transfection, as observed by the high level of EGFR knock down through ASOND therapy. Thus, ASOND-loaded NAC-C and NAP-C nanoparticles seem to be good candidates for a more in-depth cancer therapy research in future experiments in vivo.

\section{Acknowledgment}

We thank the Clinical Pharmacology Department of the University Medical Center Groningen, The Netherlands for their technical support.

\section{Disclosure}

The authors declare no conflicts of interest in relation to this paper.

\section{References}

1. Yadava PK. Nucleic acid therapeutics: current targets for antisense oligonucleotides and ribozymes. Molecular Biology Today. 2000:1-16.

2. Zamecnik PC, Stephenson ML. Inhibition of rous-sarcoma virusreplication and cell transformation by a specific oligodeoxynucleotide. Proc Natl Acad Sci U SA. 1978;75(1):280-284.

3. Stein CA. Two problems in antisense biotechnology: in vitro delivery and the design of antisense experiments. Biochimica et Biophysica Acta. 1999;1489(1):45-52.

4. Israel ZH, Domb AJ. Polymers in gene therapy: antisense delivery systems. Polym Adv Technol. 1998;9(10-11):799-805.
5. Panyam J, Labhasetwar V. Biodegradable nanoparticles for drug and gene delivery to cells and tissue. Adv Drug Deliv Rev. 2003;55(3):329-347.

6. John Wiley. Gene therapy clinical trials worldwide, provided by journal of gene medicine, 2009 [online]. London, UK. http://www.wiley.co.uk/ genetherapy/clinical/. Accessed Dec 15, 2009.

7. Thomas M, Klibanov AM. Non-viral gene therapy: polycation-mediated DNA delivery. Appl Microbiol Biotechnol. 2003;62(1):27-34.

8. Dass CR. Cytotoxicity issues pertinent to lipoplex-mediated gene therapy in-vivo. J Pharm Pharmacol. 2002;54(5):593-601.

9. Lv HT, Zhang SB, Wang B, Cui SH, Yan J. Toxicity of cationic lipids and cationic polymers in gene delivery. $J$ Control Release. 200610;114(1):100-109.

10. Wagner E. Strategies to improve DNA polyplexes for in vivo gene transfer: will "artificial viruses" be the answer? Pharm Res. 2004;21(1):8-14.

11. Yarden RI, Wilson MA, Chrysogelos SA. Estrogen suppression of EGFR expression in breast cancer cells: a possible mechanism to modulate growth. J Cell Biochem. 2001;232-246.

12. McGaffin KR, Acktinson LE, Chrysogelos SA. Growth and EGFR regulation in breast cancer cells by vitamin $\mathrm{D}$ and retinoid compounds. Breast Cancer Res Treat. 2004;86(1):55-73.

13. Lee KY, Kwon IC, Kim YH, Jo WH, Jeong SY. Preparation of chitosan self-aggregates as a gene delivery system. $J$ Control Release. 1998;51(2-3):213-220.

14. Atyabi F, Talaie F, Dinarvand R. Thiolated chitosan nanoparticles as an oral delivery system for amikacin: in vitro and ex vivo evaluations. J Nanosci Nanotechnol. 2009;9(8):4593-47603.

15. Luangtana-anan $M$, Opanasopit $P$, Ngawhirunpat $T$, et al. Effect of chitosan salts and molecular weight on a nanoparticulate carrier for therapeutic protein. Pharm Dev Technol. 2005;10(2):189-196.

16. Celis J. Cell Biology. 2nd ed. USA: Academic Press; 2009.

17. Freshney R. Culture of Animal Cells. 4th ed. Wiley-Liss: USA; 2009.

18. Promega Corporation. Technical bulletin. 2006. Available from: www.promega.com.cn/techserv/tbs/TB241-550/tb317.pdf. Accessed Dec 15, 2009.

19. Jagannath C, Wells A, Mshvildadze M, et al. Significantly improved oral uptake of amikacin in FVB mice in the presence of CRL-1605 copolymer. Life Sciences. 1999 2;64(19):1733-1738.

20. Jeong YI, Choi KC, Song CE. Doxorubicin release from core-shell type nanoparticies of poly(DL-lactide-co-glycolide)-grafted dextran. Arch Pharm Res. 2006;29(8):712-719.

21. Tondelli L, Ricca A, Laus M, Lelli M, Citro G. Highly efficient cellular uptake of c-myb antisense oligonucleotides through specifically designed polymeric nanospheres. Nucleic Acids Res. 1998;26(23):5425-5431.

22. Carlisle RC, Etrych T, Briggs SS, Preece JA, Ulbrich K, Seymour LW. Polymer-coated polyethylenimine/DNA complexes designed for triggered activation by intracellular reduction. J Gene Med. 2004;6(3):337-344.

23. Roche Corporation. Technical bulletin. 2006. http://search.cosmobio. co.jp/cosmo_search_p/search_gate2/docs/FER_/K1621.20060615.pdf. Accessed Dec 15, 2009.

24. Miller KJ, Das SK. Antisense oligonucleotides: strategies for delivery. Pharm Sci Technolo Today. 1998;1(9):377-386.

25. Dindo D, Dahm F, Szulc Z, et al. Cationic long-chain ceramide LCL-30 induces cell death by mitochondrial targeting in SW403 cells. Mol Cancer Ther. 2006;5(6):1520-1529.

26. Lee D, Zhang W, Shirley SA, et al. Thiolated chitosan/DNA nanocomplexes exhibit enhanced and sustained gene delivery. Pharm Res. 2007;24(1):157-167.

27. Salay LC, Schreier S. Effect of a kosmotropic ion on doxorubicin selfassembly and interaction with biomimetic systems. Springer Berlin/ Heidelberg 128/2004. 2004.

28. Carlisle RC, Etrych T, Briggs SS, Preece JA, Ulbrich K, Seymour LW. Polymer-coated polyethylenimine/DNA complexes designed for triggered activation by intracellular reduction. $J$ Gene Med. 2004;6(3):337-344. 
29. Wang Q, Villeneuve G, Wang ZX. Control of epidermal growth factor receptor endocytosis by receptor dimerization, rather than receptor kinase activation. EMBO Rep. 2005;6(10):942-948.

30. Lee D, Zhang W, Shirley SA et al. Thiolated chitosan/DNA nanocomplexes exhibit enhanced and sustained gene delivery. Pharm Res. 2007;24(1):157-167.

31. Ozbas-Turan S, Akbuga J, Sezer AD. Topical application of antisense oligonucleotide-loaded chitosan nanoparticles to rats. Oligonucleotides. 2010;20(3):147-153.

32. Manchanda R, Nimesh S. Controlled size chitosan nanoparticles as an efficient, biocompatible oligonucleotides delivery system. J Appl Polym Sci. 2010;118(4):2071-2077.
33. Azizi E, Namazi A, Haririan I, et al. Release profile and stability evaluation of optimized chitosan/alginate nanoparticles as EGFR antisense vector. Int J Nanomedicine. 2010;5:455-461.

34. Bordelon H, Biris AS, Sabliov CM, Monroe WT. Characterization of plasmid DNA location within chitosan/PLGA/pDNA nanoparticle complexes designed for gene delivery. J Nanomater. 2011;8:1-8.

35. Gazori T, Haririan I, Fouladdel S, Namazi A, Nomani A, Azizi E. Inhibition of EGFR expression with chitosan/alginate nanoparticles encapsulating antisense oligonucleotides in T47D cell line using RT-PCR and immunocytochemistry. Carbohydr Polym. 2010;80(4):1042-1047.
International Journal of Nanomedicine

\section{Publish your work in this journal}

The International Journal of Nanomedicine is an international, peerreviewed journal focusing on the application of nanotechnology in diagnostics, therapeutics, and drug delivery systems throughout the biomedical field. This journal is indexed on PubMed Central,

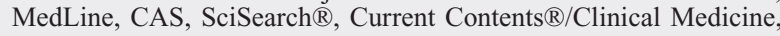

\section{Dovepress}

Journal Citation Reports/Science Edition, EMBase, Scopus and the Elsevier Bibliographic databases. The manuscript management system is completely online and includes a very quick and fair peer-review system, which is all easy to use. Visit http://www.dovepress.com/ testimonials.php to read real quotes from published authors.

Submit your manuscript here: http://www.dovepress.com/international-journal-of-nanomedicine-journal 